Voix et Images

volxetimages

\title{
Victor-Lévy Beaulieu écrivain professionnel
}

\section{Jacques Pelletier}

Volume 3, numéro 2, décembre 1977

Victor-Lévy Beaulieu

URI : https://id.erudit.org/iderudit/200102ar

DOI : https://doi.org/10.7202/200102ar

Aller au sommaire du numéro

Éditeur(s)

Les Presses de l'Université du Québec

ISSN

0318-9201 (imprimé)

1705-933X (numérique)

Découvrir la revue

Citer ce document

Pelletier, J. (1977). Victor-Lévy Beaulieu écrivain professionnel. Voix et Images, 3(2), 177-200. https://doi.org/10.7202/200102ar d'utilisation que vous pouvez consulter en ligne.

https://apropos.erudit.org/fr/usagers/politique-dutilisation/ 


\section{Victor-Lévy Beaulieu écrivain professionnel}

\section{LA FILIATION LITTÉRAIRE: FERRON}

J. P. Hugo, Joyce, Kerouack, Lowry, Melville sont vos écrivains préférés. Au Québec, vous ne semblez reconnaître que Ferron. Que trouvez-vous chez Ferron que vous ne trouvez pas chez les autres?

V.-L. B. Disons que si j'avais à faire un choix de mes écrivains québécois, il y aurait, outre Ferron, Gabrielle Roy que je considère comme le romancier le plus populaire dans le sens noble du mot, et au XIXe siècle, si on commence par le début, il n'y en a pas beaucoup, deux en fait, Louis Fréchette et Faucher de Saint-Maurice, celui-ci plus important que Fréchette à certains points de vue; au $\mathrm{XX}^{\mathrm{e}}$ siècle il y a bien sûr Louis Hémon, puis ensuite Gabrielle Roy, Ringuet, Jacques Ferron.

Pourquoi Ferron? Parce qu'il y a tout chez lui. C'est à mon avis, l'écrivain le plus important que le Québec a jamais produit. Pour toutes sortes de raisons, c'est le seul écrivain qui connaisse vraiment le pays dans tous les sens du mot. C'est le gars qui connaît aussi bien la toponymie du pays que les patronymes du pays, que la petite histoire du pays, la littérature du pays et sa petite littérature, c'est le seul gars qui m'a vraiment passionné pour une raison bien simple: sa curiosité. Ce que je reproche à bien d'autres écrivains, c'est leur absence fondamentale et totale de curiosité.

Le seul qui soit allé en profondeur dans ce qu'on est vraiment, c'est Jacques Ferron. Jacques Ferron qui a été assez astucieux et habile pour incorporer à son œuvre des éléments très modernes, sans que ça paraisse trop trop. Je lis depuis deux ans le Ciel de Québec et je m'amuse à retrouver les sources du roman; je me suis rendu compte qu'il a dû dévorer juste pour le Ciel de Québec deux cents ouvrages absolument pas connus, comme la Vie de Marie-Rose d'Eulalie Durocher dont à peu près personne ne sait qui elle est: la fondatrice du couvent de Longueuil, etc. Ce que je trouve passionnant, intéressant, c'est tout ce savoir de Ferron, qui n'est pas de l'érudition mais un savoir lié directement à la recherche littéraire comme telle. Quand tu lis les Contes du pays incer- 
tain, tu découvres qu'il a lu beaucoup de contes, qu'il connaît sa job de conteur, et quand je lis d'autres romanciers québécois passionnés de leur métier, je constate qu'ils n'ont pas été aussi loin que Ferron, qu'ils n'ont pas approfondi assez leur métier pour lui faire donner autre chose que des œuvres de reproduction.

Pour moi, la différence entre un roman intéressant et celui qui ne l'est pas, c'est ce qu'il ajoute à un ensemble culturel. Depuis cinq ans j'ai lu l'ensemble de la production culturelle québécoise au niveau du roman. et je n'ai pas trouvé chez les jeunes romanciers un seul livre qui aille à la cheville de la Famille Plouffe de Roger Lemelin, ce qui est tout dire. Ferron, lui, nous apprend quelque chose que je n'ai pas appris des autres, de là sans doute ma passion ferronnienne.

J.P. Ne considérez-vous pas tout de même que d'autres écrivains sont importants, à d'autres points de vue, je songe à un Aquin par exemple?

V.-L. B. Oui, mais quand je considère toute l'importance qu'on accorde aux autres par comparaison à celle qu'on accorde à Ferron, je trouve ça ridicule. Aquin, c'est sûr que c'est un romancier très important. Mais entre lui et Ferron, mon choix est très simple. Aquin, c'est peut-être le premier romancier véritablement urbain que le Québec ait produit. Ferron lui dit qu'il est le "dernier notable». On pourrait dire qu'Aquin est le “premier citadin ». L'œuvre d'Aquin est moderne, véritablement moderne, la première que le Québec ait produite. C'est une œuvre urbaine, ce n'est pas une affaire de campagne, ce n'est pas une affaire de village, c'est une affaire du monde moderne. Mais, d'un autre point de vue, je continue à penser que Ferron, c'est plus important. Prochain Épisode et Trou de mémoire sont de grands romans, meilleurs sûrement que la majeure partie de la production française. Bien entendu, ils n'ont pas percé à l'étranger. Pour toutes sortes de raisons qu'on connaît. J'ai toujours prétendu, pour ma part, qu'un succès à l'étranger d'un auteur québécois, c'était un succès de malentendu, un succès de folklore. Aquin n'était pas un auteur folklorique, il ne pouvait donc pas avoir de succès là-bas. Si tu regardes les œuvres qui ont eu du succès, c'est MarieAgelas, Une saison qui est une œuvre anachronique. Qu'on l'ait préférée à Prochain Épisode en 1965, je trouve ça symbolique.

Pour en revenir à la comparaison Aquin-Ferron, je dirais qu'à certains points de vue ils se ressemblent. Aquin était un gars brillant. II connaissait les nouvelles structures, il a inscrit ses romans dans une perspective carrément moderne. Ferron, lui, fait du rapiécage, du fignolage, du bricolage mais je prétends qu'avec tout son savoir il n'a pas su écrire de véritables romans. Le Ciel de Québec, par exemple, c'est une chronique. II y a tout là-dedans pour faire un excellent roman, mais il manque une synthèse des événements qui y sont, des anecdotes, qui aurait permis de produire un véritable roman. Aquin, lui, maîtrisait ça. Il pouvait faire un roman tout à fait moderne, où pour la première fois le Québec était sur le même plan que l'Italie ou le monde latin ancien. $A$ ce niveau- 
là, il n'avait pas de problèmes. Ce qui a fait de ses œuvres des livres pas toujours faciles à lire pour le commun des mortels.

En fait, si j'avais trois auteurs à donner pour la période contemporaine, il y aurait Ferron, Aquin et Ducharme, mais Ducharme pour une raison bien précise et bien stricte, le langage dont il est un magicien. Le reste, ça me paraît peu intéressant: les histoires d'enfants, pour ma part, ça ne m'a jamais intéressé outre-mesure.

\section{II. ÊTRE UN ÉCRIVAIN PROFESSIONNEL}

J.P. En dix ans, vous vous êtes révélé comme l'un des écrivains les plus prolifiques au Québec, - une dizaine de romans déjà, des textes de théâtre, des essais, etc. - comment expliquez-vous cette rage d'écrire?

V.-L. B. J'écris beaucoup, sans doute, mais je ne suis pas le seul. J'en connais d'autres. Je pense à un gars comme Jean-Claude Germain, par exemple, ou à Michel Garneau. Il y a peut-être une notion qui a changé. Jusqu'à tout récemment l'écrivain québécois était un écrivain du dimanche, mais non un écrivain professionnel dont la première fonction dans la vie est d'écrire. Moi, je me considère comme un écrivain professionnel, c'est-à-dire que je passe tout mon temps à écrire et à nourrir cette écriture-là. Or ça change peut-être pas mal des écrivains qui ont une autre job qui les emploie à plein temps et qui écrivent durant leurs loisirs un livre tous les deux ou trois ans. Or moi je prétends qu'un écrivain doit l'être complètement, c'est-à-dire à plein temps. Tu dois avoir tout tọn temps. D'abord pour écrire. Ensuite pour alimenter cette. écriture-là, parce que ça aussi c'est un problème dans la littérature québécoise, t'as beaucoup d'écrivains, beaucoup de gens qui écrivent, mais peu d'écrivains véritables. Puis il faut pas se faire d'illusions, écrire un premier roman c'est assez facile: tu pars de tes tripes, et ça va, mais si tu en écris d'autres, il faut que tu les alimentes, que tu trouves le temps de lire, et c'est ce qui fait la différence. Dans ce sens-là, je prétends, moi, que je n'écris pas tellement.

J. P. II reste qu'au Québec c'est assez rare que quelqu'un écrive autant que vous.

V.-L. B. Oui. Mais c'est peut-être poser la question du mauvais bout, ou y répondre du mauvais bout. En fait c'est pas nécessairement mauvais qu'un écrivain écrive un livre tous les deux ou trois ans, mais moi j'ai besoin de plus d'espace. Et plus j'écris, plus je me sens porté à écrire. Habituellement, tous les ans en mai je n'écris pas, je prends des vacances d'écrivain. Et en juin c'est parfois difficile de recommencer, parce que tu deviens paresseux facilement. Mais une fois la machine en marche, une fois que c'est parti, plus j'écris plus j'ai l'intention d'écrire et plus j'ai des projets. Je ne sais pas comment d'autres écrivains fonctionnent. J'imagine qu'ils travaillent seulement en fonction du livre qu'ils écrivent à l'instant présent, que leur livre n'amène à rien 
d'autre. Moi, quand j'écris un roman, cela en suscite d'autres et si je m'écoutais j'en ferais plusieurs en même temps, et je n'arrêterais jamais.

J.P. Comment se pose le rapport de l'écriture des livres d'une part et de la production de type journalistique, dont vous vivez surtout j'imagine?

V.-L. B. Oui, j'ai vécu de ça pendant deux ans à peu près. Au Québec, j'ai l'impression que c'est plus facile qu'ailleurs de faire ça. Un jour, Jacques Ferron m'a dit quelque chose que j'ai trouvé fabuleux: un écrivain doit avoir une politique, comme un gouvernement. Ferron me parlait alors de la sienne, que j'avais trouvée assez géniale. A un moment donné, je devais faire du journalisme pour gagner ma vie. Et je me suis demandé: quel travail va me permettre d'avoir plus de temps pour écrire et me permettre de traiter de sujets qui m'intéressent et qui me serviront éventuellement pour mes romans? C'est comme ça que j'ai fait du journalisme à Perspectives. Tu soumets tes projets de reportage qui sont acceptés ou non. La plupart des sujets que j'ai soumis m'intéressaient, pas tellement du point de vue journalistique, que parce qu'ils me permettaient d'entrer dans des mondes où je n'étais jamais allé, par exemple écrire un article sur Guy Lafleur ça me permettait d'entrer en contact avec le monde du Forum, de voir des joueurs de hockey, de connaître un monde inconnu pour moi. Au Devoir c'était différent. Ce que je trouvais intéressant, c'était d'écrire sur des sujets qui viennent d'arriver, sur lesquels on n'a pas encore réfléchi.

J. P. Ça alimente aussi, d'une certaine façon, la réflexion sur l'écriture.

V.-L. B. La chance que je trouvais là-dedans, c'est que l'un nourrissait l'autre. Au Devoir, je parlais surtout de livres que j'avais lus par intérêt bien plus qu'en tant que journaliste obligé de lire quelque chose qui ne l'intéresse pas, par métier. Le problème, c'est que parfois ça prenait beaucoup de temps. Les Descriptions générales de la Nouvelle France par Charlevoix, par exemple, tu te tapes ça durant une semaine, ça te donne cinquante dollars...

J.P. Je voudrais reformuler ma question d'une autre façon: on peut, c'est sûr, vivre d'une écriture polyvalente, mais peut-on vivre seulement d'une écriture dite littéraire au Québec; autrement dit, écrire, au sens plein du mot, peut-il être un métier ici ?

V.-L. B. Je ne pense pas, en effet, qu'au Québec on puisse vivre vraiment de son métier d'écrivain. Moi, je considère que je suis avant tout un romancier et je ne peux pas vivre de mes romans. Les seuls écrivains qui pourraient vivre de leur plume, ce sont ceux qui écriraient en même temps pour la télévision. C'est le seul débouché qu'un écrivain a. La production journalistique, non plus, on ne peut pas se faire d'illusions. Tu écris un texte pour Perspectives, à tous les quinze jours ou trois semaines, tu ne peux pas vivre de ça. Quant aux droits d'auteurs, moi, l'an dernier, avec quatorze livres sur le marché, j'ai fait sept cents dollars. Un article dans Perspectives, ça donne en moyenne deux cent cinquante 
dollars. Il faudrait en faire un par semaine pour vivre de ça. Et Perspectives ne peut pas en prendre un par semaine. De sorte que tu es obligé de faire cinquante-six métiers, cinquante-six misères... En plus de ça l'an dernier, je donnais un cours de littérature à l'École nationale de théâtre. Au bout du compte, tu arrives, l'un dans l'autre. Mais c'est tellement des petites choses parcellaires... Moi j'ai vu la différence lorsque je travaillais aux Éditions du Jour et aux Éditions de l'Aurore. A l'Aurore, je travaillais douze heures par jour, six jours par semaine. Mais c'était quelque chose de régulier. Chaque semaine tu étais payé, même si le salaire n'était pas énorme. Pigiste, c'est plus incertain, tu te demandes chaque semaine si tu auras un contrat, tu cherches continuellement de nouveaux sujets à proposer, et finalement ça ne t'aide plus, le quotidien passe en premier, et l'essentiel au second plan.

J. P. Mais l'écriture, comme métier, - et Ferron là-dessus disait, si je me souviens bien: l'écriture n'est pas un métier, n'importe qui peut écrire - qu'en pensez-vous? Personnellement, je me sens assez d'accord avec Ferron là-dessus. Comment pourrait-il y avoir une corporation, ou un syndicat d'écrivains? Dans la mesure où l'écrivain est un être à part - le musicien, lui, apprend la musique, fréquente des écoles de musique, sait jouer ou non, est rémunéré pour ça - ne fréquente pas d'école d'écriture, n'apprend pas vraiment à écrire de façon scolaire et n'est pas breveté comme tel. L'écrivain, autrement dit, peut-il être défini comme un véritable «professionnel » au même titre qu'un autre, comme le médecin l'est, comme le plombier l'est, etc.?

V.-L. B. Moi, je suis bien d'accord avec Ferron qui dit que l'écriture est l'utilisation de son droit de parole et que tout le monde, à la limite, peut être écrivain. Mais une union des écrivains, je me suis demandé à quoi ça correspondait. J'ai fait ma petite enquête pour me rendre compte que finalement une union des écrivains ne peut que perpétuer un privilège que l'écrivain a toujours eu et je me suis demandé: c'est quoi la fonction sociale de l'écrivain? Dans le passé, c'était simple: il s'agissait de glorifier le régime en place. Ç'a toujours été ça et les écrivains n'ont pas eu de problèmes tant que cette fonction sociale-là a été bien claire et bien identifiée. Ça remonte loin: Gengis Khan sauvait deux races de monde: les savants et les écrivains, - les savants pour les machines de guerre, les écrivains pour sa gloire... - cette situation a duré longtemps, jusqu'à la période industrielle où il y a eu un changement: la fonction sociale de l'écrivain a changé, mais les pouvoirs, eux, sont demeurés libéraux, ils ont continué à subventionner les écrivains - avant, les média subventionnaient les écrivains, maintenant, ce sont les pouvoirs publics qui les subventionnent - et pour les mêmes raisons de prestige, bien que ce soit inutile aujourd'hui. L'écrivain est poigné là-dedans et il se demande: c'est quoi, ma fonction sociale?

Personnellement, j'ai l'impression que je n'ai plus de fonction sociale en tant qu'écrivain puisque la fonction traditionnelle n'existe plus. Alors, ce n'est pas facile. Actuellement, il me semble qu'il n'y a pas de fonction 
sociale pour l'écrivain. II n'y a pas de fonction tout court, pour l'écrivain. Je ne me fais pas d'illusions: je suis lu par quatre cents personnes, peut-être cinq cents. On dit: ça se répercute dans le temps, dans vingt ans je serai encore lu c'est sûr, mais si je continue à écrire, c'est parce que j'aime ça, c'est sûrement pas parce que je compte faire de l'argent avec ça un jour - ça ne va même pas tellement ensemble: écrire et faire de l'argent:- l'idée qu'écrire soit monnayable correspond à une vision carrément traditionnelle, libérale de l'écrivain et dans ce senslà les unions des écrivains... Remarque que j'ai lu le manifeste de l'Union des écrivains à laquelle on ne m'a pas invité - et en lisant ça, je me suis dit: c'est une union carrément petite-bourgeoise; une union des écrivains comme il aurait pu en exister il y a deux ou trois cent ans; maintenant, ce n'est plus défendable: on ne fait pas une union des écrivains pour négocier un nouveau contrat d'édition; qu'est-ce que ça va changer?

J.P. Ça ne changera pas grand-chose, car de toute façon les gens n'en vivent pas.

V.-L. B. Que les électriciens s'unissent, c'est d'accord, ça va de soi. Mais que les écrivains qui n'ont pas nécessairement de liens entre eux finalement, à tous points de vue, fassent une union: pour quoi faire? Tu fais une union pour négocier un pouvoir économique. Si tu lis le manifeste de l'Union des écrivains, tu te rends compte qu'il s'agit de pouvoir négocier de meilleures subventions aux pouvoirs publics, ça va se limiter à cela. Je trouve ça un peu ridicule pour ce que ça va donner puisque ça ne changera rien à la base, étant donné que l'écrivain d'ici ne vit pas de ça. Dans un sens, je me dis même que c'est tant mieux qu'il ne vive pas de ça, parce que ne vivant pas avec ça, il est poussé par autre chose que l'argent à écrire.

J.P. Vous avez déjà dit que vous étiez un plagiaire. Comment intégrezvous le matériel des autres, comment plagiez-vous et, question subsidiaire, comment travaillez-vous?

\section{V.-L. B. Ça dépend des livres.}

Pour la Nuitte de Malcomm Hudd, je suis parti d'une phrase dite par un de mes oncles qui prenait un coup solide - il pouvait commencer à boire à dix heures le soir et le lendemain matin à onze heures il buvait encore et parlait toujours -; une fois il avait dit: "pourquoi ils riaient tous de moi parce que je faisais rentrer mon cheval dans la maison ": cette phrase m'a trappé, je suis arrivé chez moi et j'ai écrit cette phraselà. Je suis parti de cette phrase-là pour écrire le roman, sans savoir ce que cela allait devenir, et puis au fur et à mesure qu'il s'est écrit, il a pris de nouvelles directions. Ça, c'est pour.la Nuitte de Malcomm Hudd.

Pour les Grands-Pères, c'était tout à fait différent. C'était à l'époque où Jean-Guy Pilon était monté sur ses grands chevaux et commandait des textes, à Radio-Canada, pour faire des radio-romans. On m'a de- 
mandé de présenter un projet. Ce devait être très court, une histoire d'une quarantaine de pages. Je me suis installé à ma table et j'ai commencé à écrire l'histoire prévue pour Radio-Canada: ce qui se passe dans un magasin général de village avec trois ou quatre vieux qui jouent aux cartes. Une fois le travail commencé, je me suis dit: que le diable emporte Radio-Canada et le projet a pris une tout autre direction. J'ai écrit quatre histoires parallèles: une qui était l'enfance du vieux, l'autre sa vieillesse, sa dernière journée en fait, l'autre l'histoire de ses femmes. J'ai écrit les quatre histoires en parallèle. Une fois que ce travail a été fait, j'ai pris les ciseaux, j'ai effectué des coupures, j'ai fait-mes liens, mes raccords. Et ça a donné les Grands-Pères.

Pour Don Quichotte de la démanche, je suis parti de l'idée d'un écrivain qui prenait un coup solide et qui se mettait à écrire un roman, mais je ne savais pas ce que j'allais mettre dedans, la seule chose que je savais c'est que je voulais trente-trois chapitres. Une lubie comme ça, car je ne savais pas comment j'allais m'organiser pour y arriver. Je me suis mis à écrire le livre et ça a pris cette forme-là, et tranquillement ça s'est fait.

Pour en venir au plagiat, je dirai que j'ai été frappé par une phrase de Faulkner qui disait que l'écrivain est un être profondément amoral, qui devait prendre son bien là où il le trouvait sans se gêner. Je me suis dit: c'est pas bête: le gars qui monte une locomotive ou un tracteur, il se sert de tout ce qui a été fait avant lui et il ne se gêne pas pour incorporer ça à son invention; pourquoi l'écrivain ne ferait-il pas la même chose? S'il trouve une phrase ou une idée intéressante ailleurs, pourquoi ne pas l'incorporer à ce qu'il fait, sans plus. Dans Don Quichotte, je me suis bien amusé à le faire. Pour passer d'un chapitre à un autre par exemple, je me servais d'une belle phrase de Virgile - que je lisais à ce moment-là, car je lis toujours beaucoup lorsque j'écris - qui faisait le lien. Maintenant je pense que le plagiat peut être plus subtil. Par exemple, les critiques ont dit que je m'étais inspiré d'Au-dessous du volcan de Lowry pour le Don Quichotte. Moi, ça m'a fait bien rire, car la grande inspiration de ce roman-là, c'est le livre de Virgile. Le plagiat, c'est dans ce sens-là que j'utilise ce mot-là et je ne vois pas pourquoi on aurait plus de respect qu'il faut pour la littérature qui a déjà existé, par exemple pourquoi ne pourrais-je pas m'approprier le Don Quichotte de Cervantès?

J. P. Votre réponse me renseigne sur la genèse de vos romans, sur les circonstances de leur venue au monde, mais ne $m$ 'indique pas comment vous travaillez. Je reformule donc ma question: travaillez-vous à la façon d'un Butor par exemple avec des plans, des schémas ou encore à la façon d'un Zola avec des fiches, une documentation, des plans très élaborés? Sinon, comment?

V.-L. B. Je n'emploie pas de système de fiches, même quand j'écris un essai. J'utilise des plans très sommaires. Je prétends, comme Blaise 
Cendrars, qu'un livre doit trouver sa substance alors qu'on l'écrit. Je pars d'une idée, ou surtout d'une phrase. Le plus important, pour moi, c'est de trouver la première phrase, celle qui commence le livre.

J.P. Au niveau des chapitres, procédez-vous de la même façon?

V.-L. B. Dans le premier temps que j'écris, c'est une question qui ne se pose pas. J'écris tout à la main avec des stylos-feutre - sinon je n'écris pas -, trois heures par jour, six jours par semaine, sans faire de paragraphes et de chapitres. J'écris mon histoire de la première à la dernière ligne, sans me relire, sans faire de ratures, parce que je n'aime pas les ratures, ça m'agace. J'ai une écriture très petite: une page à la main, ça me donne trois pages et demie à la machine. Avant comme je n'aimais pas les ratures, j'avais l'habitude de prendre ma page et de la jeter à la poubelle et je recommençais. Maintenant je n'en fais plus; j'ai pris I'habitude de mettre ce que je veux mettre sur ma page. Dans un premier temps, j'écris donc tout mon texte d'un bloc, d'un jet, sans paragraphe; une fois que c'est écrit, je me relis, je rature, je fais des changements et je ré-écris tout ça moi-même à la machine à écrire dans un premier temps, puis je fais des corrections majeures sur mon manuscrit dactylographié.

J. P. Est-ce que ce travail-là est important? Y-a-t-il beaucoup de coupures, d'élagage?

V.-L. B. Oui, oui. Je retape mes manuscrits environ trois fois avant que ce soit terminé. Par exemple, le premier jet d'Un rêve québécois avait quatre cent pages. Je l'ai ramené à cent soixante pages et je l'ai changé complètement après le premier jet. Dans certains cas, les changements sont moins importants. Mais, en général, entre la première version et le manuscrit définitif, ce n'est plus du tout le même livre.

J.P. Le travail de finition, c'est donc vraiment une étape seconde, bien distincte de la première, qui est l'écriture en un seul jet?

V.-L. B. Le premier jet, il faut le faire le plus rapidement possible. C'est important: tu respires ton livre, tu es pris avec, tu n'as pas de recul. Moi, dans un premier temps je ne veux pas de recul car c'est trop aliénant, tu n'es jamais satisfait de la phrase écrite, de la situation, et si tu te remets à reprendre la situation tout de suite, tu bloques si bien que maintenant quand je bloque sur une situation ou sur un personnage que je n'arrive pas à cerner, je saute, je continue sans même faire de coupures dans mon texte, je laisse ma phrase en plein milieu et je saute à l'autre, quitte à revenir dessus par la suite. Souvent, quand tu écris, il y a des choses que tu.n'arrives pas à résoudre et après, en relisant, en reprenant ton manuscrit, tu trouves la solution sans trop de problèmes. Personnellement, j'aime bien la seconde phase: reprendre mon roman, couper dedans, réécrire, je ne trouve pas ça difficile comme travail; ce qui est difficile, c'est de concevoir la structure, les situations, les personnages; une fois ça fait, c'est «jouissif " de travailler là-dessus, 
de reprendre des passages, de passer une journée à fignoler quatre pages, juste pour t'amuser à rendre les quatre pages comme tu veux les rendre.

J.P. Il s'agit là vraiment d'un travail de seconde étape.

V.-L. B. Oui. Moi, tout ce que j'ai avant, c'est une ligne bien mince. Ainsi dans Blanche forcée, il y avait deux ou trois choses que je voulais mettre, et que j'ai mises en l'écrivant, sans savoir à priori comment elles allaient $y$ entrer. C'est après le premier jet que je m'arrête et que je me pose des questions sur ce que j'ai fait. En cours de travail, je ne veux pas me poser de questions.

\section{L'ÉCRITURE COMME PAÉOCCUPATION ET COMME THÉMATIQUE}

J. P. Ferron disait: pour être écrivain, il faut être un peu vaniteux. Encore que cette raison m'apparaît assez limitée, on peut être flatté au début j'imagine, mais après dix ans...

V.-L. B. Ça plafonne vite en tout cas. Entre nous, au Québec, il n'y a rien pour exciter bien gros ta vanité.

J. P. Mais alors, pourquoi écrire? Pour le plaisir de raconter une histoire, comme d'autres font du bricolage.

V.-L. B. Moi, je situe ça au même niveau. Dans Race de monde déjà, je me disais qu'il n'y avait pas de différence entre celui qui écrivait et celui qui fait des tours Eiffel avec des bâtons de «popsicle ": fondamentalement, ça correspond au même besoin qui s'exprime différemment. La façon de créer est différente, mais pour moi, à la limite, ça revient à la même chose, et le gars qui fait des tours Eiffel, il met toute son énergie à faire ça. Le gars qui construit des châteaux avec des capsules de Seven-Up, c'est la même chose. La différence est une différence de niveau.

J.P. Oui, mais il y a aussi autre chose. Dans le cas du livre, une autre personne est impliquée: le lecteur. À la limite, on pourrait écrire et laisser son livre dans un tiroir. Si tu publies, il y a des raisons. La vanité, dont parlait Ferron. Et sans doute un besoin d'avoir un contact - médiatisé bien sûr - avec quelqu'un. Est-ce que l'écriture est pour vous un moyen d'atteindre un lecteur et de viser à une certaine forme d'action?

V.-L. B. Je trouve que c'est compliqué de répondre à ça. C'est des questions qu'on se pose quotidiennement, mais on ne trouve pas de réponses. Ou plutôt tu trouves des réponses, mais pas satisfaisantes. Tu peux te dire: j'écris parce que j'aime ça et que je veux communiquer mes affaires à d'autres. Le problème, pour moi comme écrivain, est le suivant: d'une part il y a la vie, le mouvement, de l'autre la fixation de ce mouvement sur du papier. C'est mort d'une certaine façon et tu t'interroges: pourquoi je fais ça? Et toutes les réponses que tu apportes sont insatisfaisantes et tu continues pareil. Et tu te demandes: pourquoi je continue? 
Et ça non plus tu ne peux pas y répondre. Bien sûr, tu ne fais pas ça pour vivre avec ça. Moi, je n'en vis pas et je considère que c'est ce que je fais de plus important et que tout le reste est accessoire. Bien sûr, Goethe et Melville disaient: il n'y a pas d'indépendance, de loisir et d'écriture possible si tu n'as pas l'argent pour le faire. Par définition même, quelqu'un qui écrit, c'est un petit-bourgeois qui dispose de loisir pour écrire. Moi, j'ai l'impression que je n'écrirais pas plus que maintenant même si j'avais toutes mes journées libres. Ce qui changerait, ce serait l'orientation du reste: les lectures que j'aimerais faire et que je ne fais pas parce que je n'ai pas le temps, l'approfondissement de mon monde - ce qui te permet d'avoir du recul.

J.P. Je reviens au lecteur. Aquin, là-dessus, disait: ce que je veux, c'est faire partager au lecteur mon bonheur d'écrire. $Y$ pensez-vous, vous, au lecteur non pas nécessairement au moment de l'écriture, mais avant ou après?

V.-L. B. Quand j'écris, j'essaie de ne pas y penser. Parce que je prétends qu'écrire c'est d'abord une affaire entre soi et soi.

\section{J.P. Mais après, une fois le livre sur le marché?}

V.-L. B. Une fois le livre sur le marché... ? Je lisais l'autre jour une entrevue de Roger Fournier qui disait recevoir beaucoup de lettres. Moi, depuis que j'écris, j'en ai reçu peut-être deux ou trois, de bêtises d'ailleurs. J'ai donc très peu de feed-back de lecteurs.

J.P. Ça ne veut pas dire que vos livres ne sont pas lus ni discutés. Moi, «lecteur professionnel par mon métier, j'écris rarement à un écrivain...

V.-L. B. C'est sûr, qu'une fois ton livre sur le marché, tu es un peu curieux de savoir qui te lit. Au départ, je suis conscient que je ne m'adresse pas à une masse de monde. Je n'ai pas l'impression que je fais des livres pour tout le monde. De toute façon, la littérature de masse, ça se fait de moins en moins et ce sera de moins en moins possible d'en faire. De plus en plus, tu t'adresses à des publics "spéciaux", à des fragments de public. Et il n'y a qu'une exception, c'est le grand roman descriptif qui, lui, s'adresse encore à une masse de gens. Si tu ne fais pas de grands romans descriptifs, - remarque que j'aimerais en faire un, qui concilie ce que je veux faire et les goûts, les attentes des lecteurs, mais personne au Québec, n'a encore réussi ça...

J.P. C'est aussi ce que je pense. J'ai, pour ma part, un côté alukacsien » qui me fait aimer des productions comme celles de Dos Passos par exemple: il y a dans ce genre de roman la description d'une société à travers des destins individuels privilégiés. C'est personnellement la sorte de romans que j'aime.

V.-L. B. Je suis convaincu qu'il se publierait ici un grand roman descriptif que ce serait un succès. Le problème, à l'heure actuelle, c'est que le grand roman descriptif est niaiseux. De l'autre côté, les bons écrivains... 
J. P. ...ont tendance à faire de la littérature de bocal. Je pense à un roman comme celui de Rivard, Mort et naissance de Christophe Uiric, c'est brillant, mais ça me semble très «littéraire», enfermé dans un univers très clos.

V.-L. B. C'est la question que je me pose. Comment faire l'alliance sacrée entre le roman descriptif et le roman que j'appelle de recherche? Pour que tout cela corresponde à la sensibilité du lecteur. Et ce n'est pas facile. Mais on pourra peut-être y arriver. Je prétends qu'Ulysse de James Joyce, ce pourrait être un grand roman populaire. Mais ce ne l'est pas. Or ç'aurait pu l'être car c'est un grand roman descriptif. Ce qui me fascine dans Ulysse, c'est ça. Je regarde ça, en lecteur professionnel, et je me dis: ce pourrait être un grand roman descriptif populaire. Quand on aura trouvé le joint entre ça et l'autre roman, on aura une littérature...

J.P. Mais il faut faire un acte de foi, car on n'a pas l'impression que ça avance beaucoup depuis trois ou quatre ans. On a eu une première «belle période» dans l'après-guerre avec Gabrielle Roy, Ringuet, puis une deuxième dans les années 1965-1966, et depuis on a eu quelques romans intéressants mais dans une production globale assez faible. Et on a l'impression que seuls les anciens persévèrent - Thériault, Ferron, etc. - ; les jeunes romanciers, qui ont plus de quatre livres, sont rares...

V.-L. B. On peut apporter plusieurs réponses à ça; j'ai l'impression qu'un écrivain, ici, fait un premier roman: il est jeune, il n'a pas de responsabilités, - André Major me disait: je me suis mis à taper, j'ai fait un livre et les gens, dans la rue, me demandaient: qu'est-ce que tu prépares d'autre, André? et je me suis rendu compte que j'étais un écrivain - une fois son livre fait, il en fait un ou deux autres, et il se dit: c'est pas intéressant, ça paie pas, et il se marie, a des enfants, doit se trouver une job pour vivre, et il laisse tomber l'écriture. Ceux qui ne laissent pas tomber cherchent la bonne planque, - j'ai dit un jour qu'on avait une littérature de pions d'école, de professeurs d'une part, et que d'autre part, ceux qui n'étaient pas professeurs étaient à Radio-Canada dans des jobs de bonne planque: réalisateurs, etc. La génération de la bonne planque, qui existe encore, est bien là: il y a des écrivains qui publient et dont on ne parle pas; quand il s'agit d'un gars de la génération de la bonne planque, il va passer à Radio-Canada, on va lui faire des émissions spéciales, il va avoir la première page du Cahier Arts et Lettres de la Presse et trois articles... la génération de la bonne planque, c'est ça...

J.P. Je voudrais aborder la question de la forme de vos romans. La forme du monologue, de l'écriture en spirale est caractéristique de votre écriture : pourquoi emprunter cette forme-là?

V.-L. B. J'aurais de la difficulté à donner des raisons là-dessus...

J. P. Marcotte dit dans son livre le Roman à l'imparfait - qui porte sur Godbout, Ducharme, Marie-Claire Blais et je ne sais plus qui ${ }^{\uparrow}$ - que les 
romans québécois d'aujourd'hui sont tous différents du grand roman classique, tel que Lukacs notamment le définissait; cette constatation me paraît vraie, mais il prétend qu'il n'est plus possible d'écrire aujourd'hui de grands romans réalistes comme on en a fait au XIX et au $X X e$. Moi, je m'interroge là-dessus car je me dis qu'un projet comme l'histoire des Beauchemin, ça implique peut-être une sorte de retour à cette forme de romanesque-là.

V.-L. B. Que ce ne soit plus possible, j'en doute. Mais que ça ne corresponde plus à la sensibilité de maintenant, j'en suis à peu près certain. Ces grands romans classiques-là ont été remplacés aujourd'hui, pour une bonne part, par la télévision et le cinéma. Le cinéma et la télévision et d'autres média ayant pris la relève du grand roman classique - avec description de personnages, recherche psychologique, longues descriptions -, ce n'est plus possible de faire ça aujourd'hui dans le roman moderne, quoique je suis convaincu qu'un roman comme ça, écrit et publié, serait un succès. Les grands romans descriptifs, aujourd'hui, ça marche encore très bien. J'en ai lu. Je me suis demandé pourquoi un livre comme Papillon par exemple était un best-seller. Et je me suis dit: ces romans-là vont exactement dans le sens de ce que les gens voient à la télévision. Les gens qui écrivent aujourd'hui, pour la plupart, n'ont pas cette sensibilité-là, ne pensent pas comme ça: ils font des recherches au niveau des structures, du langage romanesque lui-même et ces recherches excluent carrément le grand roman classique qui ne correspond plus au niveau de sensibilité des gens qui écrivent.

J. P. De ce point de vue-là, il reste qu'on peut aussi se poser des questions en voyant l'évolution du nouveau roman par exemple. Au début, le nouveau roman français était intéressant, mais rapidement, ça s'est mis à tourner en rond.

V.-L. B. Ç'a tourné très rapidement en rond. Le nouveau roman français, notamment, a foiré, est tombé pour une raison pas toujours bien identifiée, qui est à mon avis la suivante: ces romans manquaient joyeusement d'imaginaire. En privilégiant la structure, l'écriture a eu pour conséquence de faire perdre le reste. Dans le cadre français, ça avait un sens. Leur univers imaginaire, de toute façon, est bloqué depuis un bon moment. Tu lis Le Clézio par exemple, c'est une belle machine, - je lis ça, moi, pour ce que j'appelle la musique de l'écriture - mais pour le reste, il n'y a pas grand chose là-dedans, pas d'univers imaginaire.

Ici, tu es poigné dans cette contradiction-là: d'un côté, à cause de ce qui se vit politiquement, tu te dis: il y a un univers imaginaire; d'un autre côté, un certain nombre d'écrivains québécois connaissent les techniques nouvelles, les maîtrisent, si bien que l'écrivain québécois est pris dans une contradiction: d'un côté la structure romanesque nouvelle, de l'autre un univers imaginaire qui existe, mais qu'il n'a pas appro- 
fondi, qu'il connaît mal; ça produit les romans qu'on connaît, les miens y compris.

C'est pas toujours facile de faire le joint. Les Sud-Américains, eux, et c'est ce qui m'intéresse dans leurs œuvres, ont réussi à faire le joint. Quand je lis l'Obscène oiseau de la nuit de José Donoso, je me dis: ce gars-là a tout lu, il a lu Joyce et les autres, il les a assimilés. Je me dis: comment font-ils pour assimiler, intégrer tout ça et produire des œuvres profondément originales? Ce qui ne m'apparaît pas le cas ici. Je lisais récemment l'entrevue d'un auteur qui disait: la littérature québécoise existe, c'est beau, etc. Je regrette, mais ce n'est pas vrai: la littérature québécoise, pas plus que l'américaine, pas plus que n'importe quelle autre, n'est plus belle qu'il faut. On est tous dans le même bateau, et ce n'est pas plus gai qu'il faut. La question, ce n'est plus: peuton écrire un roman? $\mathrm{C} a$, on le peut, et on le sait...

J. $P$. La production, depuis quelques années, on a l'impression qu'elle baisse de qualité, d'une année à l'autre. On sait qu'il s'agit d'un phénomène mondial. Cette année, particulièrement, au Québec, c'est faible. Rien de très intéressant n'est sorti...

V.-L. B. Oui, et tu te dis, ça correspond à quoi ? Et je reviens à mon article dans le Devoir. Tu as tout à coup le parti québécois au pouvoir et tu constates que rien dans la fiction québécoise des dernières années n'annonçait ça. Tu te demandes: est-ce le politique qui est «way-out», ou l'écrivain qui est complètement à côté de la «traque"? J'ai lu tout ce qui s'est fait dans la production depuis cinq ans et mon point de vue c'est que ce n'est pas seulement très pauvre, mais affligeant: tu lis l'Embarquement pour Anticosti, des affaires de même... Tu penses à la production des années 1940, la Famille Plouffe, Bonheur d'occasion, il y a quelque chose tout de même là-dedans. Aujourd'hui, la littérature québécoise se divise en deux catégories bien distinctes: celle de ceux qui écrivent des romans comme on en faisait dans les années 1920 , sans même les "recettes" qu'on pouvait utiliser en 1920 et, de l'autre côté, celle des gens qui font des recherches formelles, langagières; ces deux mondes sont très coupés l'un de l'autre et on n'arrive pas à voir le lien entre ça.

J.P. C'est ce qui me frappe aussi. Et c'est très limité dans les deux cas. D'un côté, c'est limité sur le plan formel. De l'autre, c'est limité parce qu'il n'y a pas grand-chose. Et parfois la mécanique même ne fonctionne pas très bien. Plusieurs romans sont intéressants plus au niveau du projet qu'à celui de la réalisation. Je songe à un roman comme le Noyau de Desruisseaux, par exemple...

V.-L. B. C'est un phénomène courant. Je pense à un autre cas, dans une autre veine, Johnny Bungalow de Paul Villeneuve. Je trouve que le projet est intéressant, mais raté au niveau de l'exécution parce que le gars écrit mal, fait penser des gens de 1930 comme on pense aujourd'hui. En 1970 ou 1972 - je ne sais pas quand au juste il a été écrit - je 
trouve qu'au plan formel ça ne tient pas, ce n'est pas logique, c'est incohérent comme discours. Mais le projet était mauditement intéressant. Un des bons projets des dernières années...

J.P. Personnellement, je serais moins sévère dans ce cas-là; je trouve qu'il y a des erreurs - le prologue notamment, les notes, etc. - mais dans l'ensemble je trouve ça bon...

V.-L. B. La question est la suivante: ces romans-là ne sont pas "professionnels". II faut bien dire qu'il y a tout un travail au niveau de l'édition qui ne se fait pas. Le manuscrit de Villeneuve, je l'avais lu quand j'étais au Jour. Ma suggestion c'était que le roman soit coupé de moitié: il aurait été bien meilleur. Il ne manque pas grand-chose à ce livre-là pour être un grand livre. Mais ce n'est pas là... Remarque que je ne trouve pas la production française plus glorieuse pour autant mais ce n'est pas le problème...

J.P. Jusqu'à Blanche forcée y compris, vous annonciez un livre: Sur la littérature. Ce projet n'apparaît plus dans N'évoque plus que le désenchantement... Pourquoi? Parce que votre réflexion sur la littérature, elle est là?

V.-L. B. Non, ce livre-là n'est pas abandonné. Le titre, lui, est abandonné et comme je n'en ai pas trouvé un autre, j'ai arrêté de l'annoncer, mais c'est un livre que je fais à compter de l'automne et qui va porter en particulier sur les littératures sud-américaines. Je prends les littératures sud-américaines un peu comme témoins de la littérature et je vais en parler d'une façon particulière: il ne s'agira pas d'un essai comme tel, mais de la lecture que je fais de ces œuvres-là, et à travers cette lecture, passera une réflexion plus générale sur l'écriture. C'est loin donc d'être un projet abandonné; j'ai maintenant la documentation qu'il me faut, et je m'y mets à compter de septembre.

J.P. En fait, il s'agira donc d'un projet très différent de celui de N'évoque plus que le désenchantement..., d'un ouvrage moins personnel, plus objectif, impliquant une prise de distance par rapport à l'écriture.

V.-L. B. Dans N'évoque plus que le désenchantement, il s'agit d'un point de vue bien personnel. Dans le livre Sur la littérature, je parlerai de la question des littératures nationales; qu'est-ce qu'une littérature nationale, comment ça naît, qu'est-ce que ça fait en prenant pour exemples des gens comme Asturias, Borges, Pardiso, Donoso, etc. II s'agira de se demander quels sont les points de ressemblance, de convergence entre la production sud-américaine et la production québécoise. Quand tu lis des romans sud-américains, tu n'es pas dépaysé. Je me demande: comment arrivent-ils, les Sud-Américains, à écrire des livres comme ceux-là alors que nous, on n'y arrive pas? Ça me fait me poser toutes sortes de questions non abordées dans N'évoque plus que le désenchantement. 
J.P. La réflexion sur l'écriture est très importante dans votre œuvre, surtout depuis Oh Miami... et le Don Quichotte au point de constituer un des thèmes majeurs de l'œuvre. Un privilège est accordé à l'écrivain et ce depuis le début d'ailleurs, déjà dans Race de monde on a affaire à un héros écrivain - et la question qu'on se pose c'est: pourquoi cette valorisation de l'écrivain, pourquoi ce type de héros plutôt que d'autres?

V.-L. B. En fait, c'est parti d'une drôle d'affaire. Dans Race de monde, je voulais planter un décor, une mise en scène. Mon intention était ensuite d'écrire un livre sur chacun des personnages présentés. Et puis je ne l'ai pas fait, parce qu'à un moment donné ça ne me tentait plus de le faire. Puis je me suis rendu compte que finalement on parle toujours de soi, des nombreux soi qu'on porte. Alors je me suis dit: pourquoi ne pas admettre d'emblée que je suis au centre de cette affairelà ? A ce moment-là, l'écrivain devient un personnage plus important que les autres, privilégié parce que c'est de lui que tout part. Mais à partir de N'évoque plus que le désenchantement, la réflexion là-dessus, je pense, est pas mal terminée: l'écrivain n'est pas plus intéressant que qui que ce soit.

J. P. Don Quichotte de la démanche marque chez vous une étape et une crise; c'est le roman de l'interrogation sur l'écriture. La réponse semble plutôt négative dans le Don Quichotte: l'écriture éloigne de la vie, elle ne libère pas, ne guérit pas, ne règle rien, elle essaie de dire la réalité du monde, mais elle n'y réussit pas: Abel, sur le plan de sa pratique d'écriture, semble en crise et pourtant vous écrivez toujours et Abel Beauchemin aussi... N'évoque plus que le désenchantement c'est un peu la contrepartie positive du Don Quichotte. Les impasses de l'écriture sont moins soulignées et on trouve même un écho de ce que Durrell écrivait dans le Grand Suppositoire, je crois: écrire est facile, mais vivre est moins drôle. Est-ce que le Don Quichotte, dans votre œuvre, marque vraiment une crise comme, en tant que lecteur, on peut le supposer?

V.-L. B. Curieusement, non. If faudrait peut-être d'abord situer ça. Le point de départ est assez curieux. Un jour, à un lancement aux Éditions du Jour, Jacques Benoît parlait d'écriture et, à un moment, il a dit: un problème va se poser un jour pour nous autres, il va falloir trouver un second souffle, il va y avoir une étape difficile à franchir. Moi, je trouvais ça un peu bizarre. Je ne m'étais jamais posé la question. Une fois chez moi, je me suis dit: ce ne serait peut-être pas une mauvaise idée de faire un roman qui porterait là-dessus, qui serait une espèce de réflexion... Je suis parti de ça. Mais, en écrivant Don Quichotte, c'était plutôt le courant contraire du livre qui m'animait; j'étais plutôt "peppé", je m'amusais bien gros en le faisant, tandis que N'évoque plus que le désenchantement ç'aurait plutôt été le contraire: c'est plutôt là que je me demandais vraiment, étant donné mes expériences d'édition, si ça valait vraiment la peine, et j'étais prêt à mettre au pilon à peu près 
tous les écrivains que j'avais rencontrés, que j'avais connus, pour les avoir vu vivre un peu, pour avoir connu un peu mieux leurs idées, ce qu'ils pensaient: si c'était ça, les écrivains, ça ne valait vraiment pas la peine, l'écriture était vraiment le dernier métier qu'on devrait faire, la dernière fonction à avoir un sens dans le monde.

J.P. C'est curieux car, du point de vue du lecteur, le Don Quichotte apparaissait comme une interrogation personnelle par personnage interposé bien entendu sur l'écriture...

V.-L. B. Non, dans Don Quichotte, c'est plutôt le contraire. Quand j'ai fait ça, ce livre-là, je suis parti de l'idée de Benoît et j'ai voulu montrer que la réponse à sa question n'était pas difficile, qu'il n'y avait rien là, que des échecs, des manques mêmes on pouvait faire de la littérature avec ça. Dans N'évoque plus que le désenchantement, c'est la démarche inverse et tout ce qui sauve ma réflexion, ou ce qui la fait, c'est la passion d'écrire, peu importe le reste, et c'est là sans doute la justification de cet acte.

J.P. Au bout du compte, ce qui apparaît, c'est qu'on continue à écrire même si on n'est pas certain que ça vaut quelque chose. Dans Don Quichotte, deux ou trois questions importantes sont posées sur l'écriture et la réponse donnée est que, lorsque tu écris, tu ne vis pas. On donne toutes sortes de bonnes raisons pour ne pas écrire. Et finalement on écrit quand même...

V.-L. B. Alexandre Dumas a répondu à ça: j'écris parce que je ne sais rien faire d'autre. C'est peut-être aussi bête que ça.

\section{L'OEUVRE}

J.P. Bessette distingue dans votre production des romans "familiaux" et des romans "personnels", ce qui me semble assez juste, mais le roman «familial » n'est vraiment développé que dans Race de monde et un peu dans le Don Quichotte; autrement dit, il y a comme une renonciation du projet "familial", «social " du début - l'histoire des Beauchemin comme on dit l'histoire des Rougon-Macquart chez Zola - au fil de l'œuvre: pouvez-vous expliquer ce glissement, ce déplacement de projet?

V.-L. B. II y a eu effectivement un déplacement de projet. J'ai mis de côté Steven le Hérault, l'histoire du frère d'Abel, et les trois livres que je prévois toujours faire (la Vraie Saga des Beauchemin); ceux-là, je les ai mis de côté, non pas au niveau du travail souterrain - documentation, prise de notes, etc. - mais au niveau du projet immédiat: ce seront de gros livres - mille cinq cents pages pour les trois - et je veux pas manquer mon coup. Je calcule que pour les faire il me faudra dix ans à plein temps. Tant que je n'aurai pas mon dix ans, je ne les ferai pas. En attendant, je liquide autre chose. Mais je continue à travailler: je prends des notes, j'esquisse des croquis. La Grande Tribu, je voulais 
l'écrire à seize ans; à cet âge, je n'ai pas été capable de le faire. Mais j'y songe toujours.

En attendant, je fais autre chose. Et puis un roman en amène d'autres et certains projets sont reportés. Par exemple, il y a deux ans, j'ai écrit un roman, Blanche forcée, qui devait être un simple récit, sans plus. Et puis je me suis fait chiper mon manuscrit. J'ai dû donc reprendre le roman, et en le récrivant, j'ai essayé d'établir des points de repère, et, pour la première fois de ma vie, j'ai fait un plan, pas un plan du livre mais un plan des éléments que je voulais mettre dedans, par exemple, un langage marin - ce qui m'a conduit à des recherches un peu fofolles sur le vocabulaire marin au XIX ${ }^{e}$ siècle, qui elles-mêmes m'ont conduit à prendre connaissance du vocabulaire amérindien -, ensuite l'idée de décrire la côte sud du Québec, de Montréal jusqu'à la Gaspésie, en Indien; la terre du bout du monde. Puis je me suis dit, dans un deuxième livre, je vais exploiter la côte nord, de Sainte-Émilie-de-l'Énergie jusqu'à Pointe-Bleue, la réserve amérindienne. Là, je suis parti avec l'idée de faire deux livres là-dessus. J'ai écrit le premier, et en l'écrivant, des choses sur l'écriture me passaient dans la tête. Cela a donné N'évoque plus que le désenchantement, un entre-deux qui préparait en même temps le livre que je voulais faire sur Melville. Évidemment, c'est le Melville qui conditionnait les deux premiers. J'avais pris pour personnage un supposé océanologue qui faisait des recherches sur les baleines pour en arriver, bien sûr, à Melville. L'un dans l'autre, j'ai prévu qu'il y aurait cinq livres dans cette histoire-là. Évidemment, ça retarde autre chose.

J. P. Pour revenir à ma question, ce glissement ne signifie donc pas vraiment un abandon?

V.-L. B. Oh non, pas du tout. Je considère que mon projet essentiel, c'est celui de ma trilogie. Quand j'aurai fait celui-là...

J. P. II y a chez vous une oscillation quant à la conception et à la représentation de la société traditionnelle, valorisée dans les romans comme lieu de paix, d'harmonie et dévalorisée dans le Manuel de la petite littérature. Comment voyez-vous cette question? Comment concilier cette contradiction apparente, sinon réelle?

V.-L. B. Je pense, pour ma part, que les deux représentations sont complémentaires. Bien sûr, il y a dans mon œuvre une certaine nostalgie de la terre pour la bonne raison que le monde du petit village québécois était un monde immobile, fermé, où les choses ne changeaient pas de place: par exemple, mon grand-père, qui a quatre-vingt-seize ans, n'est jamais sorti de son village; pour lui, le monde c'était les limites de sa terre. Or évidemment, c'est très sécurisant ce monde-là; une fois les éléments mis en place par la religion, l'éducation, rien ne bouge. Si tu compares ce monde à celui du Manuel de la petite littérature, c'est sûr qu'il y a une différence. Celui décrit dans le Manuel de la petite littérature a existé aussi, mais je ne pense pas que le Québécois en général - 
sauf celui des villes qui était plus menacé, parce qu'il vivait dans un milieu qu'il ne connaissait pas, celui du curé Labelle qui disait au monde: si vous restez à la campagne, vous ne risquez pas de vous perdre, mais à la ville, c'est la promiscuité, etc., univers dont témoignent les livres dont je parle dans le Manuel de la petite litterature qui donnent une vision de la campagne du point de vue de la ville - je ne pense donc pas que le Québécois corresponde à l'image fournie par les livres consacrés aux mystiques et aux mutilés; le Québécois, bien sûr, était conservateur, ne changeait pas beaucoup, avait peur de tout parce que la religion lui disait qu'il fallait avoir peur de tout, mais je pense que l'un dans l'autre c'est complémentaire. Évidemment, mon point de vue quand j'écris un roman est subjectif; moi j'ai vécu à la campagne et j'aimais ça, la campagne, alors que le point de vue du Manuel de la petite littérature est sans doute plus objectif: le colon qui allait en Abitibi, ça devait pas être gai tous les jours, c'était même pas gai du tout.

J.P. Ça ne réfère peut-être pas à la même société traditionnelle. Celle des romans, c'est la société rurale d'avant les entreprises de colonisation, la société rurale de souche. Celle dont parle le Manuel de la petite littérature, c'est celle de la période du retour à la terre, avec tout l'appareil de propagande idéologique qui l'a accompagnée.

V.-L. B. Moi, j'ai l'impression qu'il y a eu deux mouvements. Avant 1850 , le Québécois qui vivait à la campagne n'était pas malheureux de son sort. Ça, on le retrouve dans les monographies de paroisse. A TroisPistoles, par exemple, vers 1850 , on ne faisait pas une vie trop difficile: on ne faisait pas les foins, on ramassait le foin de grève, on ne cultivait pas parce qu'il y avait du gibier. Ce qu'ils n'arrivaient pas à accepter, c'était leur isolement l'hiver. Mais, une fois qu'ils eurent trouvé des moyens de s'amuser, ils n'eurent plus beaucoup de problèmes. C'est arrivé après 1850, quand on a trouvé que les Québécois devaient être instruits, et qu'on a fait appel à un clergé conservateur et très colletmonté et qu'on a imposé aux Québécois la mentalité qu'on connaît. Avant ça, je ne pense pas que ça existait: le Québécois était un anarchiste d'abord, un individualiste - j'ai lu dans des monographies que des gars l'hiver préféraient tuer leurs vaches et garder leur cheval parce qu'ils trouvaient ça plus beau; comme aujourd'hui, à la campagne, on trouve des maisons toutes croches avec un gros «char "à la porte...

J. P. Vos héros, de manière générale, que ce soit Satan Belhumeur, Jos Beauchemin sont des petits-bourgeois révoltés, mais non des révolutionnaires. Diriez-vous qu'ils expriment, d'une certaine manière, les préoccupations de toute une génération de Québécois à la fin des années 1960 ?

V.-L. B. Peut-être. Sans doute. Pour un personnage comme Jos Connaissant, c'est assez évident. C'est le gars coupé de ses racines qui sombre dans une espèce de mysticisme dont le renouveau charismatique d'au- 
jourd'hui est un peu l'équivalent. Toute une clientèle québécoise existe pour ça aujourd'hui. Si je regarde la génération de Parti pris, avec le recul dont nous disposons, je me dis: ces gens-là étaient au fond des gens de robe. II y a trente ans, ç'aurait été des curés. Au début des années 1960, ils étaient "révolutionnaires" puis ils sont devenus ce que moi je prétends qu'ils étaient déjà, c'est-à-dire des missionnaires laïques: ils ont pris le courant de la nouvelle culture, de la contreculture... Le plus bel exemple, le cas-type c'est Chamberland. Tu prends Pierre Maheu, c'est une autre direction mais voisine, Raoul Duguay qui a été dans les derniers moments de ça, et je pense que Jos Connaissant symbolise peut-être tout ce courant-là. II a des problèmes émotifs, qui sont des problèmes qu'on retrouve dans le mysticisme importé d'un Jacques Renaud. Moi je trouve que ça va tout à fait dans ce courant-là.

J. P. C'est aussi ce qui m'a frappé en lisant ces romans-là qui résument assez bien les préoccupations de la petite-bourgeoisie intellectuelle de la fin des années 1960, début des années 70 , qui a connu la tentation politique puis s'est tournée vers les orientaleries.

V.-L. B. Quand j'ai fini le Jos connaissant, je voulais lui donner une suite. Dans le prochain roman, à paraître à l'automne, Jos revient et je pense que c'est la suite logique du mysticisme de Jos connaissant qui prend racine-là. Ce qui est arrivé aux Québécois depuis sept ou huit ans, c'est le mysticisme à l'orientale et là on assiste au mysticisme néo-chrétien où l'on ressuscite Scot Érigène et ces gens-là, et dans le roman à venir, Jos est là avec son nouvel appareillage.

J.P. Vos romans ont, en général, une forme bien précise. Réginald Martel a écrit un jour dans la Presse, avec raison, que vos romans prennent la forme de longs soliloques de personnages le plus souvent alcooliques, mystiques ou à demi-fous, à l'exception peut-être de Blanche forcée dont le personnage-narrateur tient un discours plus rationnel: pour vous, ces personnages ont-ils une dimension symbolique? Et ce choix ne vous éloigne-t-il pas d'une représentation d'ensemble du Québec (telle que pourrait la fournir un récit de l'histoire des Beauchemin)?

V.-L. B. Disons d'abord que j'ai privilégié les ivrognes peut-être parce que j'en ai connu et que j'en connais beaucoup. Et j'ai l'impression que c'est une race de monde qui n'est pas prête de mourir au Québec. Je ne sais pas: ça me paraissait important d'en parler. Ce sont des personnages dont la littérature québécoise n'a pas parlé beaucoup. Je pense, par exemple, à un personnage comme Barthélémy Dupuis dans Un rêve québécois: ce n'est pas un personnage dont on a beaucoup parlé dans la littérature québécoise.

\section{J.P. Sauf dans le Cassé de Renaud.}

V.-L. B. Oui, mais d'un point de vue assez différent. II me semble que ces gens-là symbolisent, pour une bonne part, l'état de la nation. Ce sont des asociaux bien sûr, mais j'ai l'impression souvent que le Québec est 
peuplé d'asociaux. II y aurait toutes sortes de raisons à donner pourquoi c'est comme ça. C'est une réalité qu'il m'apparaissait important d'évoquer. Comme je trouvais important de parler de la sexualité.

J. P. Vos personnages, en général, sont presque tous tournés vers le passé, nostalgiques de l'enfance: ce sont de grands enfants ou des adolescents prolongés, si l'on préfère. Êtes-vous conscient de cette constante et comment l'expliquez-vous?

V.-L. B. Que ce soit une constante, je l'admets. Que ce soit conscient, aussi. Pourquoi les personnages sont comme ça, la réponse est plus difficile.

J. P. Mais pourquoi, autrement dit, le choix de ces personnages-là?

V.-L. B. C'est un choix. Que mes personnages soient des adolescents attardés ou de grands enfants, c'est vrai, mais j'ai l'impression que la plupart des gens sont comme ça. On ne le voit pas, parce que c'est maquillé par cinquante-six bebelles, parce que les gens sont encadrés, mais on en a la preuve, dès que les gens sortent de leur cadre naturel de fonctionnement, tout saute. Ce sont les maquillages de la société qui font qu'on s'en aperçoit pas mais j'ai l'impression qu'il y a véritablement peu d'hommes et de femmes, dans le sens plein du mot, si on gratte un peu, qui ne soient pas effectivement de grands enfants.

J. P. Le thème du passé revient très souvent dans votre ceuvre. Un passé qui est, en général, valorisé dans les romans. Or cette valorisation du passé est associée à la société traditionnelle. On retrouve reprise la vieille thématique du roman québécois, pas dans les termes d'autrefois, aucun roman ne prônant par exemple le retour à la terre, mais il reste qu'il y a nostalgie du passé, et d'un passé rural...

V.-L. B. Le passé rural, pour une raison bien simple...

J. P. Au fond, presque tous les personnages sont des ruraux même lorsqu'ils vivent en ville, sauf peut-être Barthélémy Dupuis.

V.-L. B. Oui, lui, il n'a pas d'origine comme telle... Mais disons que cela relève sans doute de raisons bien personnelles. Je suis né, pas directement dans un milieu rural, mes grands-parents appartenant plutôt à la petite-bourgeoisie de Trois-Pistoles - mon grand-père jouait à la bourse et était assez riche -; ce n'est que lorsqu'il a perdu son argent, qu'il a fait de mauvaises affaires qu'on est déménagé à la campagne. Les six premières années de ma vie, finalement, je les ai passées à Trois-Pistoles dans un petit village tranquille. J'ai vécu à la campagne quatre ans et demi, cinq ans seulement à Saint-Jean-de-Dieu.

Pourquoi j'ai privilégié ça? Peut-être simplement parce qu'à la campagne j'aimais ça, d'une part. Un moment donné, je me suis demandé ce que j'aurais fait si mes parents n'étaient pas déménagés. Je me suis dit: un habitant probablement qui, un jour, à son tour, serait déménagé en 
ville, comme beaucoup. Tous les gens de ma famille, jusqu'à il y a vingt ans, demeuraient à la campagne. Ça vient sans doute de ça. Les Beauchemin, c'est une famille de la campagne qui arrive en ville: le seul qui ne vient pas, c'est le grand-père... Mais je serais d'accord maintenant pour dire que cela, c'est quasiment anachronique. C'est assez curieux car les romanciers des années 50 et 60 ont peu parlé de cette réalité, du passage de la campagne à la ville...

\section{J. P. II y a eu Gabrielle Roy...}

V.L. B. Oui, Gabrielle Roy en a parlé. Mais peu, sauf elle. Et je trouve que la littérature québécoise est toujours profondément anachronique par rapport à ce qui se vit, quand ça se vit. Pour toutes sortes de raisons, peut-être normales. Moi, par exemple, je suis arrivé à Montréal en 1957 et j'ai commencé à écrire à la fin des années 60 ; venant d'un milieu rural, cela s'explique que j'en parle dans mes livres, mais j'admets que, comparé à ce que le Québec a connu comme évolution, c'est assez anachronique par rapport à la réalité sociale: on aurait dû parler de ça dans les années $50 .$. Mais j'ai l'impression que la littérature est toujours anachronique par rapport à ce qui se vit. Le simple fait d'écrire explique ça: tu écris un roman ça te prend deux ans, quand il est fini, il est anachronique vis-à-vis de toi, et vis-à-vis le reste: ça ne peut pas finir autrement... À l'avenir, ce côté-là, dans mon œuvre, sera plutôt sur la voie de service: de nouveaux personnages s'en viennent...

J. P. Dans les Voyageries, en tout cas, l'avenir apparaît. Les personnages apparaissent aussi préoccupés par ça. De ce point de vue-là, ça m'apparaît assez nouveau par rappört aux romans antérieurs.

V.-L. B. Et d'autres personnages vont venir qui, pour la première fois, sont nés à la ville.

\section{v. LE POLITIQUe : QUÉBEC}

J.P. Straram a déjà écrit dans Chroniques: le point faible de Beaulieu, c'est le politique. Êtes-vous d'accord avec ce jugement-là?

V.-L. B. Je ne sais pas trop quoi répondre. Je ne sais pas trop d'abord ce qu'il entend exactement par politique. Mais il y a une tendance depuis quelques années à tout vouloir ramener à la politique et au soi-disant engagement de l'écrivain, et à sa partisannerie même. Pour moi, ce n'est pas primordial dans le sens où ça doit forcément sauter aux yeux, où tu as ça écrit dans le front. Maintenant, j'essaie de faire une réflexion là-dessus. Finalement, tout est politique. Puis après?

Personnellement je ne suis pas intéressé par les démarches politiques à court terme, pour des objectifs bien précis. Je pense qu'on peut être un écrivain très politique sans forcément tomber dans le quotidien de la politique. Il y a déjà un tas de gens qui font ça à longueur d'année, c'est leur job, et je ne vois pas pourquoi je le ferais à leur place. II me 
semble qu'on a une action en tant qu'écrivain qui doit aller plus loin que ça, qui doit passer dans les livres, mais je ne sais pas, c'est sans doute très simple et très compliqué à la fois: tu nais, tu vis, tu meurs, et quel que soit le régime politique, ces choses-là vont se produire quand même, si bien que certaines questions que tu te poses ne peuvent pas trouver réponse seulement par le politique.

Personnellement, je me considère comme un anarchiste; le gouvernement idéal, c'est celui qui gouverne le moins. Tes prises de position politiques sont toujours circonstantielles, ne peuvent pas être autre chose...

J.P. Est-ce que le jugement de Straram ne peut pas être alimenté dans une certaine mesure par des fragments de votre œuvre; je pense à Un rêve québécois qui a déjà été critiqué d'un point de vue politique dans Strategie, je songe aussi à des passages de Blanche forcée qui peuvent être interrogés d'un point de vue politique.

V.-L. B. Malcomm Reid l'a fait pour Blanche forcée. II a pris une phrase où le gars dit: ça ne donne rien d'aller manifester à Firestone, les vitriers sont déjà en place pour remplacer les vitres. En fait, si tu lis la phrase comme il faut, ça veut dire: pourquoi faire ça quand on sait que des gens, l'autre bord, s'attendent déjà à cette action-là, qu'ils sont prêts à y faire face, et que finalement tu vas aller perdre trois heures pour rien. II y a tout un romantisme là-dedans; et je pense que le romantisme, il serait peut-être temps qu'on l'abatte et qu'on regarde les choses en face.

J. P. Le 15 novembre, comme tout le monde, sauf exception, vous avez été heureux, j'imagine. Et puis récemment vous avez publié un texte désenchanté dans le Devoir. Pouvez-vous vous expliquer là-dessus?

V.-L. B. Le texte que j'ai fait dans le Devoir, ce que j'ai dit, qui ne me paraissait pas aussi clair à ce moment-là, mais les événements semblent devoir me donner raison, c'est que j'ai de plus en plus de méfiance envers le pouvoir politique quel qu'il soit. Et j'ai l'impression que ce que j'ai dit - et que je maintiens aujourd'hui - c'est que le gouvernement québécois actuel essaie de temporiser partout, de faire en sorte que l'événement qui l'a porté au pouvoir en novembre ne soit plus magique. $\mathrm{C}$ a, tu le sens dans tout ce qu'ils font. Par exemple, regarde ce qu'ils ont trouvé comme thème de la Saint-Jean - "J'ai la mémoire en fête" - un thème très traditionnel. Si ça avait été «J'ai le présent, ou encore j'ai l'avenir en fête", ça aurait eu de l'allure. Ça me dit, moi, tout ça, que les gens qui forment ce gouvernement-là sont des gens très traditionnels, puis libéraux dans le sens premier du terme. Et puis l'espèce de joie, l'exaltation qu'on a eue, le 15 novembre, est venue d'un malentendu. C'est ce que j'ai essayé d'expliquer. Et le malentendu se perpétue, tout le côté magique s'amenuise, tombe à rien: on en est rendu à réhabiliter Maurice Duplessis, on parle d'autonomie, de plus en plus on 
va aller vers le statu quo, vers ce que prétendait Robert Bourassa: faire l'indépendance sans la faire. Le $P Q$ est bien engagé dans cette voie-là. Mon article sur le 15 novembre disait, en gros, qu'il ne s'était rien passé.

J. P. Vous entretenez une vision du Québec assez pessimiste. Vous ne semblez guère croire que ce pays se délivrera jamais, atteindra un jour sa souveraineté politique. Vous semblez penser qu'au mieux on peut en tirer matière à une création littéraire importante (cela est clair dans oh Miami et le Don Quichotte). Mais cette création littéraire pourrait-elle, en l'occurrence, être autre chose qu'un chant du cygne, une oraison funèbre? L'Irlande a produit Joyce, mais qu'est-ce que l'Irlande aujourd'hui?

V.-. B. Dans mes mauvais jours, je ne vois pas beaucoup d'espoir à l'horizon. Quand je regarde notre situation politique, je me dis: comment peut-on en arriver à en sortir une fois pour toutes? II n'y a pas ici les éléments qui existaient par exemple en Afrique pour susciter une libération véritable. La situation québécoise, c'est essentiellement une situation de malentendu : le Québec, c'est un maudit pays bâtard, un pays où l'aliénation est tellement omniprésente et omnipuissante. La lecture des journaux, l'écoute de la télévision - par laquelle passe la plus grande partie de la fiction québécoise: dans les télé-romans - noús apprennent à quel point les choses sont maquillées: tous les téléromans par exemple tournent autour de la famille; or moi je prétends que la famille, ce n'est plus un problème aujourd'hui; même chose pour la violence: elle n'y est jamais présente, sauf sur le mode du comique. Tout ça pour en revenir à ceci : le Québec, c'est un malentendu, c'est une ambiguïté, une réalité bien équivoque. Et quand je regarde le parti québécois au pouvoir, je me dis: ça fait durer l'équivoque. C'est un pays en flottement tout le temps. Quand I'Algérie a voulu devenir indépendante, tu as eu une polarisation, les pour et les contre, tu as eu une guerre, et les pour ont gagné. Mais ici, tu sais jamais véritablement qui est pour, qui est contre, s'il se passe quelque chose ou rien. Tu as un gouvernement qui part avec tambours et trompettes, mais quand tu lis son discours, tu te rends compte qu'il tient le discours que tenait Duplessis finalement.

J. P. On sort sa statue d'ailleurs...

V.-L. B. C'est très symbolique. C'est plus que symbolique. Dans mes mauvais jours, je regarde ça, et je me dis: baptême, il y a rien à faire avec ça. Ça fait deux cents ans que c'est de même et ça va continuer pendant je ne sais combien de temps et je n'ai pas l'impression que c'est une loi sur la langue qui va changer ça. Les leviers économiques, même sous un gouvernement $P Q$, ça ne change pas, de sorte que je comprends bien, en un sens, les réticences d'une centrale comme la CSN, quoi que je ne la trouve pas habile du tout: tout porter sur le nationalisme québécois, c'est encore une erreur, parce que ça te fait oublier tout le reste et tu ne travailles pas sur le reste qui est le plus im- 
portant: changer les règles du jeu, fondamentalement. Tu t'interroges: changer les règles du jeu, ça veut dire, au niveau des entreprises étrangères, au niveau de l'économie, un paquet d'affaires... et tu te dis: peut-on le faire? Ça, c'est possible. On peut dire: on change tout ça. Mais comme on sait qu'il n'y a pas un empire dans le monde qui a laissé faire ça, tu t'interroges, tu te dis: c'est mal parti, ça fait deux cents ans que c'est bien mal parti, et dans mes jours les plus sombres, je me dis: ça ne peut pas réussir, ça ne marche pas...

J.P. Ce qu'on peut faire de mieux, c'est de la littérature... C'est un peu ce qui est dit dans Oh Miami.

V.-L. B. Oui. Dans mes mauvais jours, je me dis: ce qu'on peut tirer de mieux de ça, c'est des livres. Mais c'est dans mes mauvais jours...

Cette entrevue a été réalisée le 2 juin 1977 par Jacques Pelletier au domicile de l'auteur; le texte qu'on vient de lire en est la retranscription intégrale; seul l'ordre des questions a été modifié.

1. NDLR: il s'agit de Gérard Bessette. 